\title{
El turismo cultural y la digitalización del patrimonio en un entorno Linked Open Data: el caso de Europeana
}

\author{
Cultural tourism and heritage digitization in a Linked Open Data environment: the Europeana case
}

\author{
Xavier Agenjo Bullón (1), Francisca Hernández Carrascal (2)
}

(1) Fundación Ignacio Larramendi, c/Alenza 4, 28003 Madrid (España), xavier.agenjo@larramendi.es.

(2) Biblioteca Nacional de España, Paseo de Recoletos, 20-22, 28001 Madrid (España), mariaf.hernandez@bne.es.

\begin{abstract}
Resumen
El texto repasa los proyectos desarrollados por Europeana para mostrar el papel que la digitalización y los datos abiertos vinculados de patrimonio cultural pueden jugar en el fortalecimiento del sector del turismo cultural. Se aportan una serie de datos estadísticos básicos acerca del turismo y del turismo cultural para enmarcarlo como sector económico, y se revisan las estrategias fundamentales de las instituciones europeas sobre turismo cultural, así como el papel preponderante que le asignan a Europeana. Estas estrategias se han trastocado y acelerado en respuesta a la debacle económica provocada por la pandemia de COVID19. Se mencionan los principales planes estratégicos del Gobierno de España para el desarrollo de la economía, de la investigación e innovación y para la transformación digital de las Administraciones Públicas y el papel que en estos planes se asigna al turismo cultural y a las instituciones de memoria.
\end{abstract}

Palabras clave: Turismo cultural. Patrimonio cultural digitalizado. Datos abiertos de patrimonio cultural. Datos abiertos. Europeana.

\section{Introducción (1)}

La digitalización del patrimonio cultural de Europa es un proceso al que ha venido a poner orden el proyecto de Europeana. De hecho, uno de los mayores aportes de Europeana es el Europeana Data Model (EDM), que se está aplicando tanto en las bibliotecas como en los archivos o en los museos —dando lugar al acrónimo GLAM, es decir Galleries, Libraries, Archives and Museums. Bajo este acrónimo se agrupan también las pautas comunes con las que las galerías, las bibliotecas, los archivos y los museos están digitalizando su patrimonio y afrontan la transformación a la tecnología Linked Open Data, iniciativa a la que es frecuente referirse como LOD-LAM (acrónimo de Linked Open Data-Libraries, Archives and Museums).

Indudablemente, el patrimonio cultural digitalizado en un entorno Linked Open Data puede jugar un papel fundamental para impulsar todo tipo

\begin{abstract}
The paper reviews the projects developed by Europeana to show the role that digitization, linked open data and cultural heritage can play in strengthening the cultural tourism sector. A series of basic statistical data about tourism and cultural tourism is provided to frame it as an economic sector. The fundamental strategies of the European institutions on cultural tourism are reviewed, as well as the preponderant role they assign to Europeana. These strategies have been disrupted and accelerated in response to the economic debacle caused by the COVID-19 pandemic. The main strategic plans of the Government of Spain for the development of the economy, research and innovation and for the digital transformation of Public Administrations are mentioned, having in mind the role that these plans assign to cultural tourism and memory institutions.
\end{abstract}

Keywords: Cultural tourism. Digital cultural heritage. Cultural heritage. Linked Open Data. Europeana.

de acciones en distintos ámbitos, como por ejemplo, las industrias culturales o creativas, la educación y el turismo.

\section{El turismo cultural}

Este artículo está centrado en Europa y en Europeana, como epítome de las bibliotecas virtuales europeas. Sin embargo, dado que la Digital Public Library of America (DPLA) es un proyecto, si no gemelo por lo menos mellizo — de hecho su esquema de metadatos es casi idéntico- siempre es interesante comparar, aunque sea a vista de pájaro, cualquier materia en ambos proyectos. Así, más allá de las similitudes en el modelo de datos, ambas iniciativas difieren especialmente en la forma de afrontar su relación con la educación o con el turismo. Pues bien, la DPLA da muy poca importancia al turismo, lo cual es llamativo porque según la Organización Mundial del Turismo, Estados Unidos es el tercer destino más 
apreciado por los turistas a nivel global, por detrás de España y Francia (World Tourism Organization, 2021).

También hay que recordar que según la UNESCO España es el tercer país del mundo, después de China e Italia, con mayor número de bienes inscritos en la lista del Patrimonio de la Humanidad (UNESCO, World Heritage List Statistics).

The world's top 10 destinations receive $40 \%$ of global arrivals

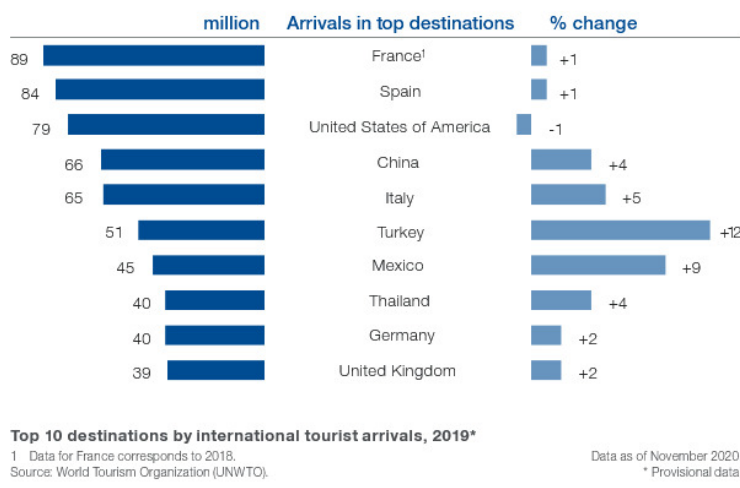

Figura 1. Principales destinos turísticos

(Organización Mundial del Turismo, 2020)

En el marco de la Unión Europea, y según las Estadísticas sobre turismo de Eurostat (departamento estadístico de la Unión Europea), el país más visitado, por el número de pernoctaciones, es España, y es también el país con mayores ingresos turísticos netos, 46.300 millones de euros en 2018 (Eurostat, 2020a). Eurostat no proporciona en estas estadísticas qué porcentaje de la actividad turística se refiere al turismo cultural.

La Comisión Europea proporciona cifras muy significativas sobre el turismo en Europa: el $40 \%$ de los turistas de la UE escogen sus destinos por razones culturales y por los sitios de patrimonio cultural. El $62 \%$ de los europeos hacen al menos un viaje de placer al año y la mayoría tiene como destino la propia Europa; el gasto en turismo durante los veranos es de 190 billones de euros; y finalmente, el $10 \%$ del producto interior bruto europeo procede del turismo.

En España, el Anuario de Estadísticas Culturales del Ministerio de Cultura y Deporte, publicado en noviembre de 2020 sobre los datos de 2019, indica que el $17 \%$ del total de viajes realizados por los residentes en España fueron iniciados principalmente por motivos culturales, mientras que el $19,8 \%$ de las entradas de turistas internacionales (14,5 millones de viajes) fue principalmente por motivos culturales (España. Ministerio de Cultura y Deporte, 2020).
Eurostat (2020b) ofrece un análisis algo más detallado en Culture statistics - 2019 edition como es el Use of information and communication technology for cultural purposes. El panorama general que reflejan las estadísticas de cultura en 2019 es que $90 \%$ de los hogares de la UE disponen de acceso a Internet. Holanda, Islandia y Noruega alcanzan el nivel más alto $(98 \%)$, cerrando la lista, Grecia (80 \%), Montenegro (80 \%) y Bulgaria (79 \%). España se sitúa en el primer tercio de la tabla con un $95 \%$.

En cuanto al uso de Internet para fines culturales (2), el análisis parte de una encuesta realizada sobre la población comprendida entre los 16 y los 74 años sobre un conjunto de actividades culturales prefijadas. Para 2019 las actividades mayoritarias son ver televisión o vídeos (70 \%), escuchar música (59 \%) y jugar o descargar juegos (33\%). El análisis ofrece también datos agregados según franjas de edad, nivel socioeconómico y sexo. (Eurostat 2020c)

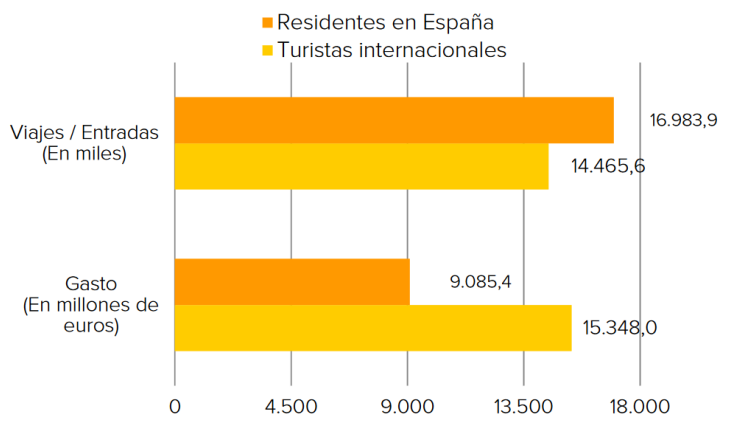

Figura 2. Viajes por motivos culturales y gasto asociado (Anuario de Estadísticas Culturales, 2020, p. 33)

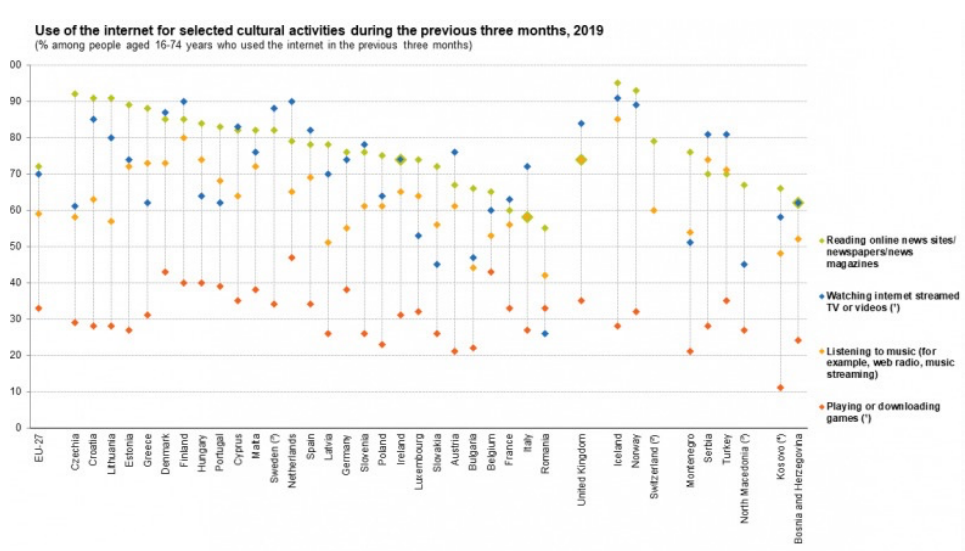

Figura 3. Uso de internet para actividades culturales (Eurostats, Culture statistics, 2019 edition)

Aunque los datos estadísticos que hemos reseñado proporcionan un panorama general sobre el 
turismo cultural o sobre el uso de Internet con fines culturales, no se recogen datos, ni puede dilucidarse de forma indirecta, el grado de influencia de bibliotecas, archivos y museos digitales en el turismo cultural. Esto nos lleva a una cuestión básica que es cómo podemos valorar, y medir, las áreas de influencia de las bibliotecas, archivos y museos digitales y de sus servicios. Y preguntarnos si es posible basar estos servicios en mediciones más o menos objetivas o, por el contrario, primero es necesario desarrollar los servicios para poder medir su impacto.

Como veremos más adelante, la pandemia ha obligado a las autoridades europeas a fortalecer el turismo cultural por medio del patrimonio digital y a acelerar la creación de servicios destinados a este sector del turismo cultural, basándose más en su urgente necesidad que en sistemas de evaluación de la gestión.

\section{Europeana: modelo de negocio y análisis de impacto}

En 2015, en el marco de la Europeana Strategy 2015-2020 (Europeana, 2017), Europeana lanzó el Europeana's Impact Assessment Framework. Su objetivo era medir su impacto y comprobar el grado de cumplimiento de los objetivos declarados en su estrategia. En 2016 Simon Tanner actualizó y ajustó este marco de trabajo e hizo algunas recomendaciones para ponerlo en práctica en el trabajo diario (Tanner, 2016).

Los resultados se presentaron en el documento MS26: Recommendation report on business model, impact and performance indicators para el que se utilizó como referencia el texto del citado Simon Tanner Measuring the Impact of Digital Resources: The Balanced Value Impact Model (Tanner, 2012). Este es un modelo teórico-práctico sobre cómo establecer las medidas e indicadores para evaluar el impacto de los recursos digitales. Registra también algunos análisis concretos de impacto económico de archivos, bibliotecas y museos, entre los que citaremos el informe final realizado por Archives, Libraries and Museums Alliance UK: Economic Impact Toolkits for Archives, Libraries and Museums: Final Report (ALMAUK, 2010). Para trasladar esta capacidad de análisis a las instituciones de memoria, en 2017 Europeana diseñó un conjunto de herramientas y procedimientos, el Impact Playbook, en el que ha participado la Impact Assessment Task Force 2.0 de Europeana (Bley, 2018).

Estos documentos no contienen instrucciones o recetas para realizar mediciones o para elaborar indicadores, directamente utilizables por los responsables de bibliotecas, archivos y museos. Además, los análisis de impacto económico que recogen se han realizado por consultoras especializadas. Se trata más bien de un conjunto de procedimientos para iniciar y configurar el proceso institucional de analizar el impacto de bibliotecas, archivos y museos, más allá de las estadísticas de visitas y visitantes a los respectivos sitios web.

Son también una muestra de la necesidad de justificar a los administradores, que controlan el reparto del presupuesto, que las instituciones de memoria son generadoras de ingresos y por tanto merecedoras de la inversión de fondos públicos. Por debajo permanece la pregunta, que quizá solo tenga una respuesta política, de si en caso de concluir en un balance negativo los archivos, bibliotecas y museos deberían dejar de proporcionar algunos de sus servicios.

En la introducción del Europeana's Impact Assessment Framework se dice literalmente:

In the for-profit world, the assessment of a good return on investment is relatively straightforward - how much did our income outweigh the costs, commonly measured in Earnings Before Interest, Taxes, Depreciation and Amortization or EBITDA. This is much harder to do in the not-for profit world where money is an input, but not the right currency for measuring output.

[En el mundo con fines de lucro, la evaluación de un buen retorno de la inversión es relativamente sencilla: en cuánto superaron nuestros ingresos a los costes, comúnmente medidos en Ganancias antes de Intereses, Impuestos, Depreciación y Amortización (EBITDA, por sus siglas en inglés). Esto es mucho más difícil de hacer en el mundo sin fines de lucro, donde el dinero es un insumo, pero no la moneda adecuada para medir la producción.]

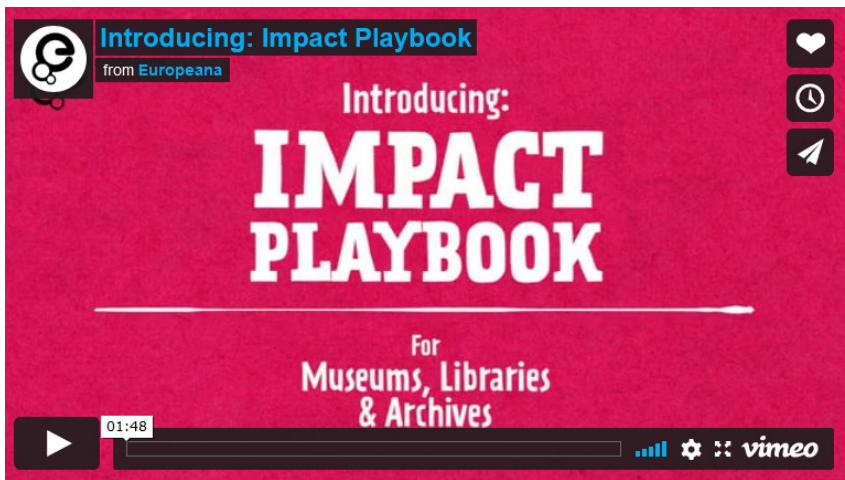

Figura 4. Uso de internet para actividades culturales (Eurostats, Culture statistics, 2019 edition)

Sea como sea, la Europeana Strategy 2015-2020 perfiló e hizo público el modelo de negocio de Europeana, modelo que tuvo su precedente en la propia solicitud de continuación de financiación 
bajo los términos de la Connecting Europe Facility, a la que se incorporó la valoración socioeconómica realizada ex profeso por la agencia SEO Economic Research (Europeana, 2017):

The model is based on the principle that cultural heritage should be freely available to end-users and that Europeana needs to develop services that generate income from three different sources: the cultural sector and the Member States that are part of our Network (service fee), the Creative Industry (revenue) and the European Commission (Connecting Europe Facility and project funding from other programmes such as Horizon 2020 and Creative Europe).

[El modelo se basa en el principio de que el patrimonio cultural debe estar disponible gratuitamente para los usuarios finales y que Europeana necesita desarrollar servicios que generen ingresos de tres fuentes diferentes: el sector cultural y los Estados miembros que forman parte de nuestra red (tarifa de servicio), la Industria Creativa (ingresos) y la Comisión Europea (Mecanismo Conectar Europa y financiación de proyectos de otros programas como Horizonte 2020 y Europa Creativa).]

Europeana ha ido construyendo ese modelo de negocio mediante un servicio central formado por los metadatos, el contenido y la tecnología disponible para las instituciones miembro; una capa de acceso a los datos en la cual es primordial el uso de Linked Open Data; y una tercera capa de servicios destinada a tres grupos principales de usuarios, los usuarios finales, los creativos y los profesionales.

En concreto, Europeana identificó distintos grupos de usuarios para los cuales la plataforma ofrecía grandes ventajas: las galerías, bibliotecas, archivos y museos; el público en general; los turistas; las industrias creativas; y las instituciones educativas y los investigadores.

En cuanto al turismo cultural, la idea subyacente en la Europeana Strategy 2015-2020 es que mejorar el acceso a la información sobre arte, cultura y patrimonio aumentará el atractivo de Europa como foco turístico, especialmente para las regiones menos conocidas. La medida de estos efectos se podría calcular mediante una estimación del porcentaje de crecimiento del turismo y a través de una estimación del incremento en la duración de las pernoctaciones y gastos de los turistas.

Se puede considerar que estas son las estimaciones que mejor medirían el impacto de Europeana en el turismo cultural, pero es también patente la dificultad de obtener estos datos, máxime si Eurostat (es decir, los Estados miembro) no incluye a Europeana como uno de los usos de Internet con fines culturales. Por otra parte, se observa un contraste entre el modelo de negocio de Europeana, y las razones que aducen las instituciones de memoria europeas, uno de sus grupos de usuarios principales, para proporcionar el acceso digital a sus colecciones.

Estas razones se prefijaron en el diseño de la última encuesta realizada por el ENUMÉRATE Observatory (3) en 2017 con los siguientes resultados:

\begin{tabular}{lc}
\hline Uso & Puntuación \\
\hline Uso académico & 8,5 \\
\hline Uso educativo & 8,5 \\
\hline Reducir el uso de los originales & 7,6 \\
\hline Disfrute personal & 6,3 \\
\hline Uso y reutilización creativos & 5,3 \\
\hline Uso ideológico, religioso o conmemorativo & 4,7 \\
\hline
\end{tabular}

Tabla I. Razones de las instituciones de memoria para proporcionar acceso digital a sus colecciones en una escala de 0 a 10

Siendo el objetivo del ENUMERATE Observatory 'proporcionar una base fiable de datos estadísticos sobre digitalización, preservación digital y acceso en línea al patrimonio cultural de Europa' (Agenjo y Campillejo, 2015) se aprecia una cierta descoordinación con la estrategia de Europeana. Parecería lógico que a la hora de diseñar la encuesta se hubieran incluido algunos de los extremos de interés para Europeana como es el turismo cultural, máxime habiéndose realizado la encuesta en el periodo en el que estaba vigente la Europeana Strategy 2015-2020.

No hay ninguna duda de que el desarrollo de servicios para el turismo cultural en Europeana es un ejemplo de cómo la plataforma ha ido evolucionando y, también, mostrando a las instituciones de memoria la forma en la que construir nuevos servicios que no estaban originalmente en la intención de los proveedores de datos.

Quizá, si tuviéramos que resumir la influencia de Europeana podríamos centrarla en la consulta multilingüe; la creación de un entorno Linked Open Data; y el desarrollo de servicios de difusión, exploración y explotación de las colecciones digitales, entre las que se encuentra el turismo cultural.

\section{Europeana y el turismo cultural}

En definitiva, para Europeana es muy importante hacer llegar al usuario de la red, que es un turista en potencia, unos mensajes basados en los contenidos digitalizados, y dado que Europeana tiene como una de sus misiones, quizá la más 
importante de todas, preservar y difundir el patrimonio, es evidente la conveniencia de establecer una línea estratégica con ese fin.

Hay que decir de entrada que la Europeana strategy 2020-2025 (Europeana, 2020) no contempla una línea específica para el turismo cultural, aunque es muy posible que tenga que replantear toda su estrategia en un escenario post-COVID-19. De hecho, la Comisión Europea ha acelerado la creación de servicios de apoyo al turismo cultural con independencia de la estrategia de Europeana. Desde luego, bien puede entenderse que los servicios de turismo cultural están contenidos en ella ya que el objetivo fundamental de Europeana, siguiendo la estrategia política de la Comisión Europea, es apoyar la transformación digital de Europa desde el sector del patrimonio cultural.

Entre las prioridades que se ha marcado Europeana están las de mejorar las capacidades de la infraestructura, mejorar la calidad de los datos, y apoyar la transformación digital de las instituciones de memoria y de los sistemas nacionales de agregación.

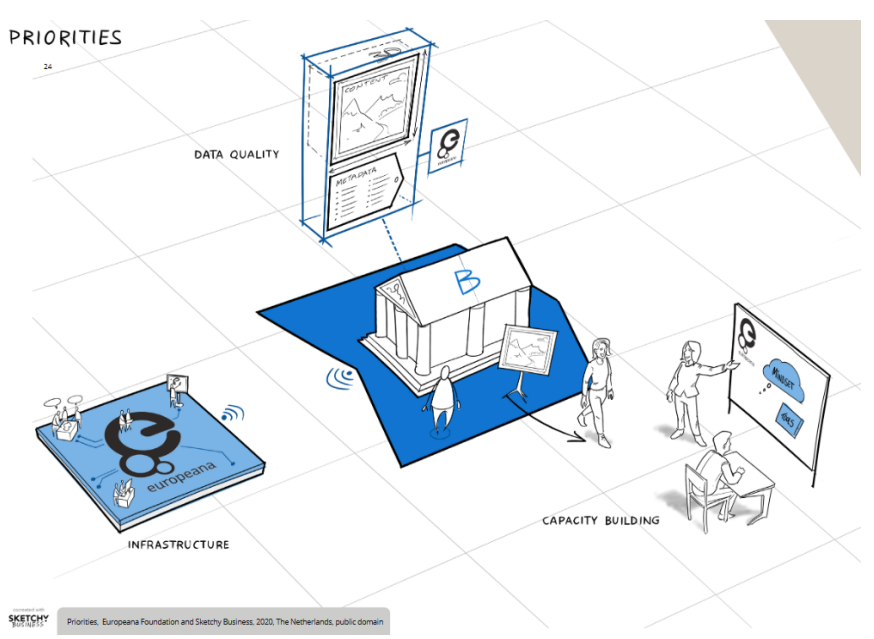

Figura 5. Prioridades

(Europeana strategy 2020-2025)

En Enhancing usability of European Digital Cultural Library using web architectures and deep learning (Machidon et al., 2020) se señala particularmente el problema de la precisión de las búsquedas en Europeana, lo que afecta también a sus API, motivada por la existencia de metadatos de baja calidad, para cuya subsanación proponen un método basado en técnicas inteligentes y de aprendizaje profundo para la interpretación, clasificación y refinado de los resultados en un motor de búsqueda amigable.

Una de las estrategias más importantes de Europeana es proporcionar datos reutilizables a terceros, lo que incluye las instituciones y empresas dedicadas al turismo. De hecho, el planteamiento de la estrategia de Europeana para 2020-2025, 'Prioridad 2 Mejorar la calidad de los datos', es muy similar a la propuesta de Machidon:

Europeana invertirá recursos en actividades relacionadas con la mejora de los metadatos y del contenido en colaboración con agregadores y proveedores de datos. Utilizará nuevas tecnologías como algoritmos de aprendizaje automático para enriquecer automáticamente o semiautomáticamente los metadatos de una manera más rápida y de forma escalable.

Pero la COVID-19 ha alterado todos los planes y estrategias y se hace imprescindible consultar la Comunicación de la Comisión al Parlamento Europeo... sobre el turismo y el transporte en 2020 y en adelante (Comisión Europea, 2020a), donde se da un análisis de la importancia del turismo para el empleo y para Europa, destacando cuáles son los puntos débiles y fuertes del turismo, y la importancia de la digitalización del patrimonio cultural. Una de las notas del texto proporciona el siguiente resumen:

El turismo cultural, que representa el $40 \%$ del turismo en Europa, está resultando particularmente afectado, ya que se han cancelado la mayoría de las actividades culturales, como ferias y festivales, e instituciones como los museos han cerrado sus puertas (92\%).

En concreto, hay una llamada a la importancia de Europeana para que contribuya al sostenimiento de este sector económico:

A partir de junio, Europeana, la plataforma europea del patrimonio cultural digital, seguirá desarrollando su vertiente de turismo, donde se muestran las joyas culturales y tesoros ocultos de Europa. En la segunda mitad del año se pondrá en marcha, en la aplicación web complementaria Cultural gems, una campaña de ciudadanos embajadores, con el fin de apoyar el turismo de proximidad.

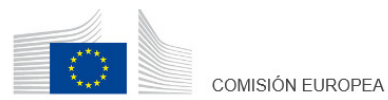

Bruselas, 29.6.2020 $\operatorname{CoM}(2020) \quad 550$ final/2

CORRIGENDUM

This document corrects document $\operatorname{COM}(2020) 550$ final of 13.05 .2020

Concerns the Spanish language version

In point III. (a), the graph on 'Tourism total contribution to GDP 2018 ' has been replaced

The text shall read as follow:

COMUNICACIÓN DE LA COMISIÓN AL PARLAMENTO EUROPEO, AL CONSEJO, AL COMITÉ ECONÓMICO Y SOCLAL EUROPEO Y AL COMITÉ DE LAS REGIONES

El turismo y el transporte en $2020 \mathrm{y}$ en adelante

COMUNICACIÓN DE LA COMISIÓN AL PARLAMENTO EUROPEO, AL CONSEJO, AL COMITÉ ECONÓMICO Y SOCIAL EUROPEO Y AL COMITÉ DE LAS REGIONES

El turismo y el transporte en $2020 \mathrm{y}$ en adelante

Figura 6. El turismo y el transporte en 2020 y en adelante (Corrección de 29 de junio de 2020) 
En este nuevo marco y con estos antecedentes, hay que mencionar que el 16 de junio de 2020 el blog de Europeana Pro publicó una entrada titulada Travelling for pleasure: a brief history of tourism (Périz, 2020), redactada por Carmen Périz Rodríguez, representante de Hispana dentro del Ministerio de Cultura y Deportes. Esta entrada contrasta con la exposición en línea titulada The Human Factor, que forma parte de la serie Heritage at Risk, donde se alerta sobre cómo la urbanización, la inestabilidad política y el turismo masificado presentan graves amenazas para los monumentos y paisajes de Europa. Seguramente, estas graves amenazas no van a ser tenidas en cuenta, al menos en un futuro próximo, dada la enorme crisis que estamos viviendo desde el año 2020.

Por esto mismo es fundamental revisar la política de la Unión Europea denominada The Europeana platform: Europe's digital cultural collection for responsible, accessible, sustainable and innovative tourism, donde ya se parte de la crisis económica que está viviendo Europa por el descenso del turismo durante la COVID-19 y se hace hincapié en el papel que debe jugar Europeana para paliar el drástico descenso del número de turistas (European Commission, 2020).

En efecto, cumpliendo con esa línea estratégica de la Unión Europea y como parte de sus iniciativas, incluidas Europe's Culture Close to you y Cultural Gems, integradas en el contexto de Reopen EU (European Union, 2020), Europeana ha creado una sección especial dentro del portal Europeana Pro titulada Discovering Europe, destinada a los profesionales del patrimonio cultural, en la que se ofrecen noticias, contenidos e iniciativas relacionadas con este sector, que ya se anunciaba en The Europeana platform... como el futuro Tourism Hub.

Es evidente, aunque no se dice públicamente, que es maravilloso conocer el patrimonio a través de plataformas como Europeana, pero que lo que de verdad se intenta es que los turistas se animen y lleven a cabo sus viajes y estancias a los lugares con alta concentración de patrimonio porque al final es lo que de verdad dinamiza la economía.

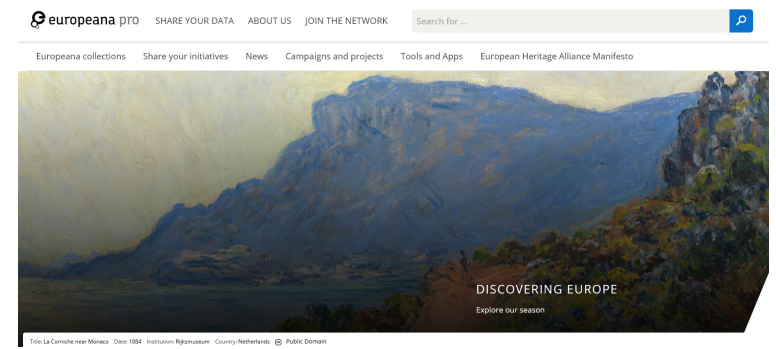

Figura 7. Discovering Europe en Europeana Pro
En definitiva, las instituciones europeas han confirmado de facto el papel de Europeana en el sostenimiento del turismo:

Europeana can inspire people to discover beautiful cities and landscapes, historic places and hidden gems across Europe. Our cultural heritage serves as the glue between the past, present and the future helping people to learn about their history.

[Europeana puede inspirar a las personas a descubrir hermosas ciudades y paisajes, lugares históricos y gemas escondidas en toda Europa. Nuestro patrimonio cultural actúa como pegamento entre el pasado, el presente y el futuro, y ayuda a las personas a aprender sobre su historia.]

También, la Comisión Europea ha movilizado a su Centro Común de Investigación para desarrollar la ya citada aplicación para dispositivos móviles Cultural Gems, que permite mostrar la riqueza e interés de las ciudades, proporcionando información de patrimonio cultural sobre OpenStreetMap.

Europeana posee una estructura de información codificada según la ontología Europeana Data Model, inmersa completamente en la tecnología Linked Open Data. Sin embargo, todavía no se está realizando la potencialidad de esas herramientas, ni su capacidad para vincular y reutilizar la información. Por ejemplo, sería fácil utilizar los vínculos entre los registros de Europeana con Wikidata y a partir de ahí acceder a la Wikipedia, que es el quinto sitio más utilizado de la Web. Wikidata tiene definida la propiedad 'Europeana entity' (P7704) para identificar personas, lugares y temas en Europeana, pero hasta la fecha sólo tienen esta relación 160.465 ítems de Wikidata.

Por supuesto sería mucho mejor que estos vínculos estuvieran reflejados en los artículos de Wikipedia, pero siempre hay un desfase entre las relaciones de Wikidata con identificadores externos y el 'control de autoridades' de los artículos de Wikipedia. A través de Wikidata y Wikipedia se podría diseñar proyectos específicos de turismo cultural. Y diríamos lo mismo de la utilización de los contenidos de Europeana en Google Knowledge Graph. De hecho, como se sabe, Google Knowledge Graph utiliza profusamente los contenidos de Wikipedia, por lo que sería factible hacer lo mismo con los contenidos de Europeana.

En cualquier caso, las líneas estratégicas y las iniciativas europeas, como Re-open EU, se refieren con insistencia a los sitios web que ha lanzado Europeana relacionadas con el turismo cultural, como Discovering Europe with Europeana Collections, y a otros como World Heritage Journeys of the European Union, The European Capital of Smart Tourism, el proyecto financiado por la Unión Europea IMPACTOUR, los European 
Heritage Awards/Europa Nostra Awards, o el European Heritage Alliance Manifesto. Como se ha mencionado, Discovering Europe ha devenido en el Tourism Hub que se anunciaba en la política de la Comisión The Europeana platform: Europe's digital cultural collection for responsible, accessible, sustainable and innovative tourism.

Además, hay que mencionar una serie de proyectos de reutilización del patrimonio por terceros, como son las apps Greetings from Zagreb, VanGoYourself, y Virtual 17th-century Flemish inn, no solamente desde el punto de vista del turismo, sino en general.

Desde luego, es encomiable la rápida respuesta para recoger las distintas experiencias que las instituciones de memoria de todo el mundo han puesto en marcha para sobrellevar la COVID-19, con las que se ha compuesto la página Creative Approaches and Collaborations de Europeana Pro, especialmente los tres finales: Collecting memories of COVID-19 in Sweden del Nordiska Museet; COVID-19 in Vienna: A collection project on the city's history del Vienna Museum; y These Times del Museum of Ordinary People. También queremos destacar un proyecto muy interesante, lanzado en estos meses de pandemia, se trata de My Home is My Museum cuyo autor son los National Museums Liverpool. En este proyecto se da mucha importancia a cómo los niños pueden ver el patrimonio, y sobre todo a las ideas que un niño puede plasmar al crear una lista de 10 objetos que puede escoger para diseñar su propia exposición. A pesar de su sencillez, incorpora más creatividad que mucho de lo que se ha hecho en este año de 2020.

Seguramente una de las selecciones más completas de portales, tours virtuales, e-learning y colecciones en línea es la recogida en el sitio web del MCN - Advancing Digital Transformation in Museums. Y, finalmente, no podemos dejar de mencionar, aunque solo sea de pasada, el esfuerzo que están realizando muchas instituciones culturales y científicas para exponer sus fondos y contenidos de una forma intensa y novedosa en el marco de Google Arts and Culture, así como la competencia que este conjunto de herramientas y app va a plantear, con toda seguridad, a Europeana.

Por nuestra parte nos gustaría mencionar el proyecto Heritage Hubs, porque guarda cierta similitud con el de la ciudad natal de los Beatles y porque en él participa la Fundación Ignacio Larramendi. Este proyecto, financiado por la Unión Europea, ha reunido a colegios de Finlandia, Serbia, Italia y España, y sus resultados se podrán ver próximamente en la Biblioteca Virtual Heritage Hubs. Muy recientemente este proyecto ha sido galardonado con el premio Europa Nostra Awards de 2021.

\section{Biblioteca Virtual HeritageHubs}

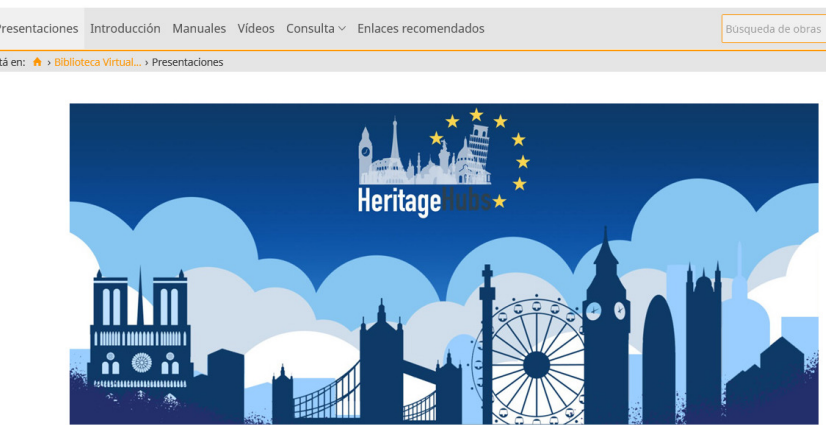

Figura 8. Biblioteca Virtual HeritageHubs

\section{Las líneas estratégicas españolas}

Es muy importante mencionar tres estrategias digitales que el Gobierno de España ha publicado en los últimos meses. Por supuesto, hay que tomar con las debidas precauciones todo lo que se está enunciando en esos tres planes porque han sido publicados en plena pandemia (cuando se revisa este artículo todavía España, y en general todo el mundo, está batallando con la tercera ola de la COVID-19).

El primer plan recibe el nombre de Estrategia Española de Ciencia, Tecnología e Innovación 2021-2027 y fue aprobada en Consejo de Ministros el 8 de septiembre de 2020 (España. Secretaría General de Investigación, 2020). Este plan menciona de pasada al turismo, englobando, y esto es muy importante, en una misma área las industrias culturales y el turismo (dentro de la sublínea Mundo digital, Industria, Espacio y Defensa de la línea Inteligencia Artificial y Robótica).

La Estrategia Nacional de Inteligencia Artificial (ENIA) (España. Gobierno, 2020), presentada el 2 de diciembre de 2020, dispone una inversión pública de 600 millones para el periodo 20212023 y supone una concreción del Plan España Digital 2020-2025, así como es el componente 16 del Plan de Recuperación, Transformación y Resiliencia de la economía española. Esta estrategia agrupa de nuevo el turismo y las industrias creativas, haciendo hincapié en que ambas constituyen un campo de cultivo para la Inteligencia Artificial, clasificándolas incluso como el sexto sector con mayor impacto esperado de la Inteligencia Artificial a corto y medio plazo, aunque no siempre queda muy claro si cuando se refiere al sector del turismo está ligado o no con las industrias creativas. 
El tercer plan, aprobado el 30 de diciembre de 2020, que lleva el membrete España Puede y el subtítulo de Plan de Recuperación, Transformación y Resiliencia (España. Presidencia del Gobierno, 2020), enuncia 10 políticas palanca de reforma estructural para un crecimiento sostenible e inclusivo. Entre ellas, y con el número 5 , se menciona una política para, entre otras cosas, la recuperación del turismo, puesto que como muy bien dice el Plan, el turismo juega un papel crucial en la economía de España y además de suponer un $10 \%$ del PIB genera importantes efectos tractores sobre otros sectores. Pero, aunque esta estrategia incluye los planes de digitalización de cuatro sectores estratégicos: salud, automoción, turismo y comercio, además del agroalimentario, no se hace una mención especial al turismo cultural, aunque sí a las industrias culturales y al turismo deportivo (Política 9 Impulso de la industria de la cultura y el deporte).

Por último, el 27 de enero de 2021 se aprobó el Plan de Digitalización de las Administraciones Públicas 2021-2025 (España. Gobierno, 2020), uno de los tres planes clave del Plan España Digital 2020-2025 junto con el Plan Nacional de Competencias Digitales y el Plan de Digitalización de Pymes. Según se anuncia, estos tres planes estratégicos dispondrán de una inversión conjunta de 11.000 millones de euros y su objetivo es reforzar la digitalización para aumentar la productividad y el crecimiento económico, impulsar la creación de empleo de calidad y conquistar los mercados exteriores. Sin embargo, no hay mención alguna al turismo, al turismo cultural, o a la cultura.

\section{Conclusión}

Este artículo, redactado en medio de una pandemia que dura ya más de un año si tenemos en cuenta la aparición de los primeros casos en España, intenta llamar la atención de los actores del patrimonio cultural, especialmente a los bibliotecarios, archiveros y museólogos, sobre el papel que pueden jugar a la hora de impulsar el turismo cultural, basándose en la estructura de información de Europeana, especialmente del uso de la tecnología Linked Open Data.

Sin embargo, a pesar del gran interés de las distintas iniciativas y aplicaciones, desde nuestro punto de vista se debería profundizar en una de las capacidades más importantes de Europeana, su entorno Linked Open Data. Únicamente la nueva app Cultural Gems se vincula con Open StreetMap, cuando es palmario que se pueden vincular muchísimos otros conjuntos de información Linked Open Data. Quizá se deba a la ur- gencia de responder inmediatamente con propuestas relacionadas con el turismo cultural para contrarrestar en algo los efectos de la pandemia.

Se puede también hacer una reflexión sobre la forma en la que la Comisión Europea y las instituciones europeas en general, ante la magnitud de la crisis, han apreciado rápidamente las posibilidades que ofrece Europeana en el sostenimiento del turismo, sin requerir la correspondiente justificación basada en detallados análisis de impacto socioeconómico, ni planes estratégicos adicionales. Además, la Comisión Europea intenta potenciar el turismo cultural aunque pueda chocar con los riesgos de un turismo masificado que se observaban antes de la pandemia.

En cualquier caso, sería una pena que no se aprovecharan las acciones que está impulsando la Unión Europea para fortalecer al turismo cultural, pero el cisne negro que está suponiendo la infección de la COVID-19 está todavía muy presente y seguro que Europeana incluirá en sus planes estratégicos esta posibilidad.

\section{Notas}

(1) Este artículo se basa en la exposición de los autores en la XXV edición de los Encuentros Internacionales sobre Sistemas de Información y Documentación, que tuvo lugar del 30 de septiembre al 2 de octubre de 2020 en la Biblioteca María Moliner de la Facultad de Filosofía y Letras de la Universidad de Zaragoza. El tema central de estos encuentros fue la relación entre las instituciones de memoria y el turismo cultural. No se le escapará a ningún lector que si este artículo se hubiera redactado antes de la pandemia de la COVID-19, el resultado habría sido muy diferente.

(2) La tablas 'Number of trips by purpose' de las Estadísticas sobre turismo de Eurostat (Eurostat Data Browser) solo recogen los siguientes motivos: Personal reasons; Professional, Business; Hollydays, Leisure and recreation; Visits to friends and relatives; y Other personal reasons. https://ec.europa.eu/eurostat/databrowser/view/tin00188/ default/table?lang=en

(3) El proyecto ENUMERATE actual tuvo su origen en un proyecto europeo denominado NUMERIC, que continuó en el proyecto ENUMERATE (2011-2014) financiado por el programa ICT de la Comisión Europea, en cuyo consorcio participó DIGIBÍS y los autores de esta comunicación. En 2014 ENUMERATE pasó a formar parte de Europeana v3, transformándose en el ENUMERATE Observatory.

\section{Referencias}

[Todos los enlaces están comprobados a fecha de 31 de enero de 2021]

About ENUMERATE. // Europeana Pro, March 24, 2015. https://pro.europeana.eu/page/about-enumerate.

Agenjo, Xavier; Campillejo, Maribel (2015). El observatorio de la digitalización en Europa. // Desafíos y oportunidades de las Ciencias de la Información y la Documentación en la era digital: actas del VII Encuentro Ibérico EDICIC 2015 
(Madrid, 16 y 17 de noviembre de 2015). Madrid: Universidad Complutense de Madrid, 2015. ISBN 978-84-6083330-7. https://eprints.ucm.es/34723/.

Archives, Libraries, and Museums Alliance UK (2010). Economic Impact Toolkits for Archives, Libraries and Museums: Final Report [2010]. https://almauk.files.wordpress.com/2010/09/alma-uk-economic-impacts-projectstage-1_final-report.pdf.

Biblioteca Virtual Heritage Hubs. Madrid: Fundación Ignacio Larramendi, 2020. http://www.larramendi.es/her_hub/es/ micrositios/inicio.do.

Bley, Jens (2018). What the Europeana Impact Playbook taught a city about revitalising an inner city square. // Europena Pro. November 5, 2018. https://pro.europeana.eu/post/what-the-europeana-impact-playbooktaught-a-city-about-revitalising-an-inner-city-square.

Byrd-McDevitt, Lori (2020). The Ultimate Guide to Virtual Museum Resources, E-Learning, and Online Collections. /I MCN Blog. March 14, 2020. https://mcn.edu/a-guide-tovirtual-museum-resources/.

Collaborative Approaches and Collaborations // Europeana Pro. https://pro.europeana.eu/page/creative-approachesand-collaborations.

Comisión Europea (2020). Comunicación de la Comisión al Parlamento Europeo, al Consejo, al Comité Económico y Social Europeo y al Comité de las Regiones: El turismo y el transporte en 2020 y en adelante. Bruselas, 13.5.2020, COM(2020) 550 final. https://eur-lex.europa.eu/legal-conte $\mathrm{nt} / \mathrm{ES} / \mathrm{TXT} / \mathrm{HTML} /$ ?uri=CELEX:52020DC0550\&from=EN

Comisión Europea. Estrategia - Prioridades 2019-2024. Una Europa adaptada a la era digital: capacitar a las personas con una nueva generación de tecnologías. https://ec.europa.eu/info/strategy/priorities-2019-2024/europe-fit-digital-age_es.

Cultural gems: Discover cultural and creative places in European cities https://culturalgems.jrc.ec.europa.eu/.

Discovering Europe. // Europeana Pro. https://pro.europeana.eu/page/discovering-europe.

ENUMERATE Observatory. // Europeana Pro. https://pro.europeana.eu/page/enumerate.

España. Gobierno (2020). Estrategia Nacional de Inteligencia Artificial. https://www.lamoncloa.gob.es/presidente/actividades/Documents/2020/ENIA2B.pdf.

España. Gobierno (2020). Plan España Digital 2025. https://www.lamoncloa.gob.es/presidente/actividades/Documents/2020/230720-Espa\%C3\%B1aDigital_2025.pdf.

España. Ministerio de Cultura y Deporte (2020). Anuario de Estadísticas Culturales 2020. https://www.culturaydeporte. gob.es/dam/jcr:52801035-cc20-496c-8f36-72d09ec6d533/ anuario-de-estadisticas-culturales-2020.pdf. Véase también el apartado Turismo Cultural del Anuario de Estadísticas Culturales 2020. Principales resultados. Página 33.

España. Presidencia del Gobierno (2020). España puede: Plan de Recuperación, Transformación y Resiliencia. Madrid, octubre 2020. NIPO: 089-20-023-7. https://www.lamoncloa.gob.es/presidente/actividades/Paginas/2020/ espana-puede.aspx.

España. Presidencia de Gobierno (2020), Plan de Digitalización de las Administraciones Públicas 2021-2025: Estrategia en materia de Administración Digital y Servicios Públicos Digitales. https://www.lamoncloa.gob.es/presidente/actividades/Documents/2021/270121-PlanDigitalizacionAdministracionesOptimizado.pdf.

España. Secretaría General de Investigación (2020). Estrategia Española de Ciencia, Tecnología e Innovación 20212017. Madrid: Gobierno de España; Ministerio de Ciencia e Innovación, 2020. e-NIPO 83120011X. https://www. ciencia.gob.es/portal/site/MICINN/menuitem.26172fcf4e b029fa6ec7da6901432ea0/?vgnextoid $=1387571 \mathrm{a} 3 \mathrm{db}$ 06610VgnVCM1000001d04140aRCRD.

Europe's Culture - close to you. https://ec.europa.eu/culture/news/europes-culture-close-you\#: : text=this\%20campaign $\% 20$ promotes $\% 20$ the $\% 20$ re, $a \% 20$ safe $\% 20$ and $\% 20$ sustainable\%20way.

European Capital of Smart Tourism: an EU initiative to reward innovative and smart tourism in European Cities. https://smarttourismcapital.eu/.

European Commission. European Commission Strategy Shaping Europe's digital future -Policies: The Europeana platform: Europe's digital cultural collection for responsible, accessible, sustainable and innovative tourism. https://ec.europa.eu/digital-single-market/en/europeanaplatform-europes-digital-cultural-collection-responsibleaccessible-sustainable-and.

European Heritage Alliance Manifesto. https://www.europanostra.org/wp-content/uploads/2020/09/20200915EHA-MANIFESTO-layout_high-res.pdf.

European Union (2020). Re-open EU [2020]. https://reopen.europa.eu/es.

Europeana (2014). We transform the world with culture: Europeana Strategy 2015-2020. [2014] https://pro.europeana.eu/files/Europeana Professional/Publications/Europeana\%20Strategy\%202020.pdf. Véase también "Europeana 2020 Strategic update". [2017] https://strategy2020.europeana.eu/update/.

Europeana (2017). Europeana DSI 2- Access to Digital Resources of European Heritage. D4.4. https://pro.europeana.eu/files/Europeana Professional/Projects/Project_list/Europeana_DSI-2/Deliverables/d4.3\%20DSI$2 \%$ 20Final\%20Project\%20Report.pdf

Europeana (2017). Report on ENUMERATE Core Survey 4. http://pro.europeana.eu/files/Europeana Professional/Projects/Project_list/ENUMERATE/deliverables/DSI-2_Deliverable\%20D4.4 Europeana Report\%20on\%20ENUMERATE\%20Core\%20Survey\%204.pdf.

Europeana (2020). Strategy 2020-2025: Empowering Digital Change. 2020. https://pro.europeana.eu/page/strategy2020-2025-summary.

Eurostat (2020). Culture statistics - 2019 Edition. https://ec.europa.eu/eurostat/statistics-explained/index.php?title=Culture_statistics_-_introduction.

Eurostat (2020). Culture statistics - use of ICT for cultural purposes. Data extracted in June 2019. https://ec.europa.eu/eurostat/statistics-explained/index.php?title=Culture_statistics_-_use_of_ICT_for_cultural_purposes.

Eurostat (2020). Estadísticas sobre turismo. Datos de enero de 2020. https://ec.europa.eu/eurostat/statistics-explained/index.php?title=Tourism statistics/es.

Eurostat (2021). Households - level of internet access (20112020). Datos actualizados a $26 / 01 / 2021$. http://ec.europa.eu/eurostat/product?code=isoc_ci_in_h\&language $=$ en $\&$ mode $=$ view .

Google Arts \& Culture. https://artsandculture.google.com/

Greetings from Zagreb. https://pro.europeana.eu/data/greetings-from-zagreb.

Heritage at Risk: Protecting and Preserving Endangered Cultural Heritage // Europeana Pro. https://www.europeana.eu/en/exhibitions/heritage-at-risk.

Heritage Hubs. https://heritagehubs.eu/es/.

The Human Factor // Europeana Pro. https://www.europea na.eu/en/exhibitions/heritage-at-risk/the-human-factor.

The Impact Assessment Task Force 2.0 // Europena Pro, January 25,2018 . https://pro.europeana.eu/project/impactassessment-2-0-task-force.

Impact: How do we know we are making a difference? // Europeana Pro https://pro.europeana.eu/page/impact. 
Machidon, Octavian; Stoica, Dragos; y Tavčar, Aleš (2020). Enhancing the Usability of European Digital Cultural Library Using Web Architectures and Deep Learning. // Cultural and Tourism Innovation in the Digital Era: Sixth International IACuDiT Conference, Athens 2019. Springer, 2020, pp.201-207 2020. https://doi.org/10.1007/978-3030-36342-0 16.

MCN - Advancing Digital Transformation in Museums. https://mon.edu/.

Museum of Ordinary People. https://www.museumofordinarypeople.com/.

National Museums Liverpool. https://www.liverpoolmuseums.org.uk/my-home-my-museum.

Nordiska Museet. Coronaviruset i Sverige - berätta för framtiden. https://minnen.se/tema/corona.

Périz Rodriguez, Carmen (2020). Travelling for pleasure: a brief history of tourism // Europeana Pro, June 16, 2020. https://blog.europeana.eu/2020/06/travelling-for-pleasure-a-brief-history-of-tourism/.

Tanner, Simon (2012). Measuring the Impact of Digital Resources: The Balanced Value Impact Model. London: King's College, 2012. https://www.kdl.kcl.ac.uk/fileadmin/documents/pubs/BalancedValuelmpactModel_SimonTanner_October2012.pdf.

Tanner, Simon (2016). Updating the Europeana Impact Assessment Framework: Recommendations on implementing the Europeana Impact Assessment Framework https://pro.europeana.eu/files/Europeana_Professio$\mathrm{nal} /$ Projects/Project list/Europeana DSI/Milestones/europeana-dsi-ms26-recommendation-report-on-business-model-impact-and-performance-indicators-2016.pdf.

UNESCO. World Heritage List Statistics. Number of World Heritage properties inscribed by each State Party. https://whc.unesco.org/en/list/stat.

UNESCO; National Geographic. World Heritage Journeys. Europe. Co-funded by the European Union. https://visitworldheritage.com/en/eu.

VanGoYourself. https://pro.europeana.eu/data/vangoyourself.

Virtual 17th-century Flemish inn. https://pro.europeana.eu/data/virtual-17th-century-flemish-inn.

Wien Museum. Corona inWien: ein Sammlungsprojeckt zur Stadtgeschichte https://www.wienmuseum.at/de/coronasammlungsprojekt.

World Tourism Organization (2021). International Tourism Highlights, 2020 Edition. Madrid: UNWTO, 2020. DOI: https://doi.org/10.18111/9789284422456.

Enviado: 2021-01-31. Segunda versión: 2021-06-06. Aceptado: 2021-06-17. 